\title{
ENFERMEDAD CARDIOVASCULAR EN PACIENTES CON ENFERMEDAD RENAL POR DIABETES
}

\author{
CARDIOVASCULAR DISEASE IN PATIENT \\ WITH KIDNEY DISEASE CAUSED BY DIABETES
}

\author{
Guillermo Dieuzeide
}

\section{RESUMEN}

La mayoría de los pacientes con diabetes e hipertensión debería tratarse con un objetivo de tensión arterial sistólica $<140 \mathrm{mmHg}$ yTA diastólica de $<90 \mathrm{mmHg}$. Menores valores de TA $<130 / 80 \mathrm{mmHg}$ pueden ser adecuados en pacientes con alto riesgo de enfermedad cardíaca y renal. Inhibidores de enzima convertidora de angiotesina (IECA) o bloqueadores del receptor angiotensina (ARA II) a dosis máxima tolerada se consideran drogas de primera línea en el tratamiento de la hipertensión arterial; en pacientes diabéticos con un índice albuminuria/creatininuria $>30 \mathrm{mg} / \mathrm{g}$ la creatinina sérica, el nivel de filtración glomerular y el nivel de potasio deben ser monitoreados. Diferentes estudios observan con estas drogas nefroprotección independientemente de la disminución de la presión arterial alcanzada. No se recomienda utilizar una combinación de IECA con ARA II. Los inhibidores de los canales de calcio son drogas seguras para el tratamiento de la hipertensión arterial en pacientes diabéticos con insuficiencia renal.

Palabras clave: enfermedad aterosclerótica cardiovascular, tensión arterial, inhibidores de enzima convertidora de angiotesina, bloqueadores del receptor angiotensina, inhibidores de canales de calcio, nefroprotección.

\begin{abstract}
Most of the patients with diabetes and hypertension should be treated with a systolic blood pressure $<140 \mathrm{mmHg}$ / and diastolic BP of $<90 \mathrm{mmHg}$. Lower levels of BP $<130 / 80 \mathrm{mmHg}$ can be adequate in patients with high risk of heart and kidney diseases. Angiotensin-converting enzyme inhibitors (ACEI) or angiotensin receptor blockers (ARA II) at maximum tolerated doses are considered first line drugs in the treatment of hypertension, in diabetic patients with a rate of albuminuria/creatininuria $>30 \mathrm{mg} / \mathrm{g}$ serum creatinine, the level of glomerular filtration and the level of potassium should be monitored. Different studies observe with these drugs, nephroprotection regardless of the reduction of attained blood pressure. It is not recommended to use a of ACEI and ARA II combination. Calcium channel inhibitors are safe drugs for the treatment of hypertension in diabetic patients, with kidney insufficiency.
\end{abstract}

Key words: atherosclerotic heart disease, blood pressure, angiotensin-converting enzyme inhibitors, angiotensin receptor blocker, calcium channel inhibitors, nephroprotection.

Revista de la Sociedad Argentina de Diabetes 2017; Vol. 51 (112-119)
Médico especialista en Endocrinología; Jefe del Servicio de Endocrinología y Diabetes Hospital Nuestra Señora del Carmen, Chacabuco Prov. de Bs. As.; miembro del Comité de Nefropatía de la Sociedad Argentina de Diabetes, Argentina

Contacto del autor: Guillermo Dieuzeide

E-mail: dieuzeideg@gmail.com
Correspondencia: San Martín 280, Chacabuco, Prov. de Bs. As., Argentina

Tel: (011) 0235-2426658

Fecha de trabajo recibido: 18/08/17

Fecha de trabajo aceptado: 30/08/17

Conflictos de interés: el autor declara que no existe

conflicto de interés

\section{Enfermedad cardiovascular}

La enfermedad aterosclerótica cardiovascular (EAC) -definida como síndrome coronario agudo (SCA), infarto agudo de miocardio (IAM), angina estable e inestable, accidente cerebrovascular (ACV) o enfermedad vascular periférica- es la principal causa de morbilidad y mortalidad en la diabetes. Las condiciones comunes de comorbilidades (hipertensión arterial y dislipidemia) son factores asociados y la diabetes confiere un riesgo independiente para el desarrollo de esta entidad.

\section{¿Cuáles deberían ser las metas de control de la tensión arterial (TA)?}

La mayoría de los pacientes con diabetes e 
hipertensión debería ser tratado con un objetivo de TA sistólica $<140 \mathrm{mmHg} /$ y TA diastólica de $<90 \mathrm{mmHg}$. Menores valores objetivos de TA $<130 / 80 \mathrm{mmHg}$ pueden ser adecuados en pacientes individuales con alto riesgo de enfermedad cardíaca y renal, si éstos no pueden ser alcanzados sin un riesgo adicional atribuible al tratamiento ${ }^{1}$ (Tabla 1).

\begin{tabular}{|l|l|l|l|}
\hline \multicolumn{1}{|c|}{ Población } & \multicolumn{1}{|c|}{ Edad $<\mathbf{5 0}$ años } & \multicolumn{1}{c|}{ Edad 50-74 años } & \multicolumn{1}{c|}{ Edad $>\mathbf{7 5}$ años } \\
\hline Población general & $<120 / 80 \mathrm{mmHg}$ & $\begin{array}{l}\text { Objetivo inicial } 140 \mathrm{mmHg} \\
\text { TTo tolerado }<130 \mathrm{mmHg}\end{array}$ & Sin datos \\
\hline $\begin{array}{l}\text { Población de alto riesgo } \\
\text { (diabetes/riesgo CV } \\
\text { incrementado y/o IRC) }\end{array}$ & $<130 \mathrm{mmHg}$ & $\begin{array}{l}<130 \mathrm{mmHg} \text { en presencia de } \\
\text { riesgo CV incrementado o } \\
\text { enfermedad renal cronica }\end{array}$ & $<140 \mathrm{mmHg}$ \\
\hline & $\begin{array}{l}<140 \mathrm{mmHg} \text { en presencia de } \\
\text { diabetes 22 }\end{array}$ & \\
\hline
\end{tabular}

Tabla 1: Objetivos de control de de la presión arterial en la población diabética y no diabética en relación a grupo etario. Adaptado de: Chobanian A. Hypertension in 2017: what is the right target? JAMA 2017; 317(6):579-580.

El Action to Control Cardiovascular Risk in Diabetes Trial (ACCORD) evaluó si establecer un objetivo de TA sistólica en $120 \mathrm{mmHg}$ era superior a establecerlo en menos de $140 \mathrm{mmHg}$ en términos de reducir infarto de mioardio no fatal, ACV no fatal y muerte cardiovascular en pacientes tipo 2 con alto riesgo de EAC. En los resultados finales, el control de la TA intensivo no demostró un descenso significativo de los eventos finales propuestos, excepto en el caso de disminución de la tasa de ACV. Este grupo tuvo el doble de eventos adversos relacionados a la medicación hipotensora, así como mayor frecuencia de hipokalemia e incremento de la creatinina respecto del control. De hecho, el NNT (número necesario a tratar) para prevenir un evento cerebrovascular fue 89 y el número necesario para hacer un daño atribuible al tratamiento fue $50^{3}$.

En un metaanálisis en adultos con diabetes tipo 2 comparando objetivos más ambiciosos de presión arterial, límite superior $130 \mathrm{mmHg}$ de sistólica y $80 \mathrm{mmHg}$ de diastólica vs objetivos standard de presión sistólica entre 140$160 \mathrm{mmHg}$ y diastólica entre $85-100 \mathrm{mmHg}$ no se encontró una significativa reducción de la mortalidad global ni de la incidencia IAM. Hubo una reducción del 35\% riesgo relativo $(\mathrm{RR})$ de presentar ACV pero la reducción del riesgo absoluto fue solo del $1 \%$ en la rama intensiva y se asoció a eventos adversos como hipotensión y síncope ${ }^{4}$.

\section{¿Cuál sería la primera droga de elección en pacientes diabéticos con hipertensión arterial?}

De acuerdo a la posición de la Asociación Americana de Diabetes (ADA) $2017^{1}$ debería utilizarse un inhibidor de enzima convertidora de angiotesina (IECA) o un bloqueador del receptor angiotensina (ARA II) a dosis máxima tolerada como drogas de primera línea para tratar la hipertensión en pacientes diabéticos con un índice albuminuria/creatininuria $>300$ mg/g (evidencia A); en rangos 29-300 mg/g se ha demostrado que dichas drogas retardan el avance a grados más avanzados del deterioro de la función renal. No debe utilizarse una combinación de IECA con ARA II (evidencia A). Si el paciente es tratado con alguno de ellos, la creatinina sérica, el nivel de filtración glomerular y el nivel de potasio deben ser monitoreados.

Una de las primeras evidencias de los beneficios de los IECA en la progresión de la nefropatía diabética fue observada por Lewis et al. ${ }^{5}$ que, al comparar pacientes diabéticos tipo 1 con albuminuria, detectaron una reducción del $48 \%$ del RR de duplicar la creatinina y una disminución del 50\% del riesgo de muerte, 
enfermedad renal terminal (ERT) y diálisis en aquellos tratados con captopril vs placebo. La reducción fue mayor en el grupo con creatininas basales superiores a $2 \mathrm{mg} / \mathrm{dl}$ (76\%). En pacientes con alto riesgo cardiovascular, que incluyeron un subgrupo importante de diabéticos, los IECA lograron una reducción de eventos macrovasculares ateroscleróticos finales y del desarrollo de macroalbuminuria en comparación a placebo, más allá de los esperados por el sólo descenso de la presión arterial $^{6}$. En 1.513 pacientes diabéticos tipo 2 con albuminuria $>300 \mathrm{mg} / \mathrm{g}$, el uso de losartán vs placebo se asoció a una reducción del $28 \%$ del riesgo de ERT para casi un mismo nivel de presión arterial medio: 142/74 mmHg placebo, 140/74 mmHg losartán7. Similares resultados se obtuvieron en pacientes diabéticos tipo 2 con irbesartán por Lewis et al. ${ }^{8}$ al lograr una reducción del $33 \%$ del riesgo de duplicación de la creatinina y una disminución del riesgo de enfermedad renal terminal del $23 \%$ en comparación a placebo. Dicha protección fue independiente de la disminución de la presión arterial alcanzada. En un estudio a cargo del Dr. Parving se obtuvo una disminución del 70\% del riesgo de aparición de nefropatía diabética clínica definida como la presencia de albuminuria superior a 300 mg/24 hs en pacientes tratados con 300 mg de irbesartán vs placebo ${ }^{9}$.

\section{¿Puede asociarse IECA con ARA II?}

Los estudios clínicos demostraron claramente que ambos tipos de drogas no deberían combinarse: en el estudio Ongoing Telmisartan Alone and in combination with Ramipril Global Endpoint Trial (ONTARGET), la combinación de ramipril y telmisartán se asoció a un incremento del riesgo de insuficiencia renal del $33 \%{ }^{10}$. Asimismo en el estudio Veterans Affairs Nephropathy in Diabetes (VA-NEPHRON) cuya población objetivo se constituyó por pacientes con nefropatía diabética avanzada (índice albumina/creatininuria >300 mg/g) demostró que la combinación lisinopril/losartán vs el tratamiento con mono- terapia (losartán) se asoció a una duplicación del riesgo de desarrollar una insuficiencia renal aguda (12,2 eventos/paciente por año en el grupo combinado vs 6,7 eventos/pacientes por año en monoterapia) y a un mayor riesgo de hiperkalemia ${ }^{11}$.

\section{¿Son equivalentes IECA con Ios ARA II en relación a la reducción de mortalidad cardiovascular en pacientes con diabetes?}

Hay pocos estudios comparativos IECA y ARA II en cuanto a mortalidad cardiovascular en pacientes diabéticos tipo 2. Un metaanálisis comparó el efecto de IECA vs placebo y/o droga activa que involucró 35 investigaciones originales (32.827 pacientes) y 13 trabajos comparando ARB2 vs no tratamiento (23.867 pacientes) y concluyó que los IECA significativamente reducen el riesgo de mortalidad global en un $13 \%$, muerte cardiovascular en un $17 \%$, eventos cardiovasculares mayores en un $14 \%$, incluidos infarto de miocardio en un $21 \%$ e insuficiencia cardíaca en un 19\%. Los ARA II, por su parte, no obtienen reducción significativa de la mortalidad global ni cardiovascular, ni tampoco reducción de eventos cardiovasculares mayores, excepto el caso de insuficiencia cardíaca (30\%). Ni los IECA ni los ARA II se asociaron a una reducción del riesgo de ACV en los pacientes con diabetes. La conclusión del mismo fue que los IECA deberían ser la primera línea de tratamiento en pacientes con diabetes tipo 2 con riesgo cardiovascular ${ }^{12}$.

No obstante, en otro metaanálisis que involucró 21.871 participantes se detectó un $10 \%$ de reducción de eventos cardiovasculares y un $17 \%$ de mortalidad cardiovascular en pacientes diabéticos tipo 2 con hipertensión arterial, considerando este grupo de drogas (IECA/ARA II) como clase ${ }^{13}$.

\section{¿Existen alternativas superadoras al uso de IECA para el tratamiento de la insuficiencia cardíaca en diabéticos?}

Recientemente un estudio que comparó el uso de enalapril 20 mg como monoterapia vs valsartán más un inhibidor de la neprilisina 
(LCZ696, denominado actualmente sacubitrilo/valsartán) en 8.442 pacientes con insuficiencia cardíaca clase II, III o IV (fracción de eyección $<40 \%$ ) demostró que esta segunda combinación se asoció a una reducción del orden del $20 \%$ de muerte cardiovascular y un $21 \%$ menos de hospitalizaciones debido a insuficiencia cardíaca descompensada ${ }^{14}$. Un 35\% de la población estudiada tenía diabetes en ambas ramas del tratamiento. Un análisis post hoc del mismo estudio sobre 3.778 pacientes diabéticos demostró que la combinación de valsartán/inhibidor de nefrilisina vs el enalapril en monoterapia redujo en $0,14 \%$ a $0,16 \%$ la $\mathrm{HbA} 1 \mathrm{c}$ en el seguimiento a tres años y al mismo tiempo disminuyó en un $29 \%$ la necesidad de emplear insulina en los pacientes con tal afección ${ }^{15}$.

\section{¿Cuándo utilizar inhibidores de los canales de calcio?}

Los inhibidores de los canales de calcio son drogas seguras para el tratamiento de la hipertensión arterial ya sea como monoterapia o en combinación. Son de elección en pacientes diabéticos, con insuficiencia renal, pacientes ancianos, de raza negra, mujeres en edad fértil, hipertensión del embarazo, hipertensión sistólica aislada y en pacientes con enfermedad coronaria estable. En combinación resultan superiores para prevenir ACV y mortalidad global, y menos efectivos para prevenir insuficiencia cardíaca.

En el estudio Avoiding Cardiovascular Events Through Combination Therapy in Patients Living With Systolic Hypertension (ACCOMPLISH) la combinación benazeprilamlodipina fue superior a la combinación benazepril-hidroclorotiazida para reducir eventos finales compuestos de muerte cardiovascular y eventos cardiovasculares. En este estudio el $60 \%$ de la población ingresada en ambos grupos tenía diagnóstico de diabetes y los niveles de presión sistólica y diastólica alcanzada fueron similares en ambos grupos ${ }^{16}$.

Asimismo en el estudio multicéntrico Anglo-Scandinavian Cardiac Outcomes Trial-
Blood Presure Lowering Arm (ASCOT-BPLA), la rama de tratamiento que incluía amlodipina/perindopril fue levemente superior a la rama de tratamiento atenolol/diuréticos tiazídicos para prevenir eventos cardiovasculares mayores, incluyendo ACV fatales y no fatales, mortalidad cardiovascular y mortalidad por todas las causas. En la segunda rama de tratamiento se observó un incremento de nuevos diagnósticos de diabetes mellitus ${ }^{17}$.

\section{¿Son los betabloqueantes equivalentes a Ios IECA y ARA II en el tratamiento de la hipertensión en la diabetes?}

En el estudio Losartan Intervention For Endpoint Reduction in Hypertension Study (LIFE) sobre 1.195 pacientes diabéticos con hipertensión arterial e hipertrofia ventricular izquierda, el losartán fue superior al atenolol y se observó una reducción del 33\% del riesgo de morbilidad y mortalidad cardiovascular, así como una disminución del 39\% de la mortalidad global con la primera droga ${ }^{18}$. Sin embargo, no se detectaron diferencias entre captopril y atenolol en el United Kingdom Prospective Diabetes Study (UKPDS) ${ }^{19}$, como así tampoco en el estudio Captopril Prevention Project (CAPPP) ${ }^{20}$.

\section{¿Son superiores los betabloqueantes con efecto alfa sobre los betabloqueantes convencionales en el control de la presión arterial en los diabéticos?}

Los betabloqueantes carvedilol y labetalol presentan un efecto vasodilatador adicional por bloqueo de los receptores alfa. Son mejor tolerados en pacientes con vasculopatía periférica, ancianos y con insuficiencia cardíaca. En el estudioThe Glycemic Effects in Diabetes Mellitus: Carvedilol-Metoprolol Comparison in Hypertensives (GEMINI) que incluyó 1.235 pacientes con diabetes tipo 2 e hipertensión, el carvedilol fue superior al metoprolol, dado que a diferencia de éste no aumentó la HbA1c, mejoró los parámetros de insulinoresistencia y determinó un descenso de la albuminuria urinaria en un $40 \%{ }^{21}$. 


\section{¿Pueden las drogas hipoglucemiantes orales reducir el riesgo cardiovascular en los pacientes con diabetes, alto riesgo cardiovascular y nefropatía diabética?}

Después del metaanálisis publicado por Nissen (2007)22, en el que se señaló que la rosiglitazona podía incrementar el riesgo de IAM en la población diabética en un $43 \%$ y el riesgo de muerte por ECV en un 64\% en comparación a tratamientos alternativos, la Food and Drug Administration (FDA) exigió a la industria farmacéutica estudios de seguridad cardiovascular para toda nueva droga; este documento dice textualmente que: "Para establecer la seguridad de una nueva terapia para tratar diabetes tipo 2, los sponsors deberían demostrar que la terapia no resulte en un riesgo cardiovascular inaceptable" 23 .

El estudio Trial Evaluating Cardiovascular Outcomes with Sitagliptin (TECOS) ${ }^{24}$ realizado en una población de pacientes diabéticos con alto riesgo cardiovascular, utilizando sitagliptina vs placebo, demostró neutralidad con respecto al riesgo de mortalidad cardiovascular. Otro estudio finalizado fue el Saxagliptin Assessment of Vascular Outcomes Recorded in Patients with Diabetes Mellitus-Thrombolysis in Myocardial Infarction (SAVOR-TIMI) en el que se evaluó la adición de la droga saxagliptina vs placebo en una población de diabéticos con antecedentes de ECV y/o múltiples factores de riesgo. Si bien el punto final primario (infarto no fatal, ACV y muerte cardiovascular) fue similar en ambos grupos al final del período de observación, en los puntos finales secundarios se observó un aumento de la internación a causa de insuficiencia cardíaca del orden del $28 \%$ en el grupo asignado a saxagliptina ${ }^{25}$.

Pese a este hallazgo, un estudio observacional realizado en Canadá que incluyó casi un millón y medio de pacientes no demostró que el uso de inhibidores de la dipeptidil peptidasa (IDPP-4) se asociara a mayor riesgo de hospitalización por insuficiencia cardíaca en comparación a otras drogas hipoglucemiantes ${ }^{26}$.

En relación a análogos GLP-1, el estudio Evaluation of Lixisenatide in Acute Coronary
Syndrome (ELIXA) utilizando lixisenatide vs placebo en pacientes diabéticos tipo 2 que habían sufrido un evento coronario hasta 180 días previos al screening, no encontró diferencias significativas entre las dos ramas en relación a los puntos finales de nuevos eventos cardiovasculares, incluida muerte de cualquier causa e insuficiencia cardíaca.

El estudio Liraglutide Effect and Action in Diabetes Evaluation of Cardiovascular Outcome Results (LEADER) incluyó 9.340 pacientes en 32 países con 410 centros involucrados con un seguimiento de 5 años, comparó el evento final primario de muerte cardiovascular, IAM no fatal y ACV en pacientes diabéticos tipo 2 con alto riesgo cardiovascular asignados a una rama tratada con liraglutide vs pacientes asignados a placebo. Los dos grupos recibieron el estándar de atención usual. El $81 \%$ de los pacientes había padecido una ECV previa y el $18 \%$ tenía múltiples factores de riesgo. Al finalizar el estudio se observó una reducción de la mortalidad cardiovascular del orden del 22\% altamente significativa, con una disminución de la tasa de infartos del $12 \%$ (NS) y de ACV del $11 \%$. En este estudio, la diferencia de $\mathrm{HbA} 1 \mathrm{c}$ entre ambas ramas fue pequeña $(0,4 \%)$ : la rama liraglutide tuvo un descenso de peso de 2,3 kg promedio con respecto a la rama placebo y hubo una diferencia de 1,2 mmHg en la presión arterial sistólica en favor de liraglutide. No se observaron diferencias en los niveles de lípidos. El otro dato significativo fue el descenso de los eventos; fue más relevante en los pacientes con clearance de creatinina $<60 \mathrm{ml} / \mathrm{min}$ vs los pacientes con clearance en rango normal ${ }^{27}$.

El segundo estudio que empleó análogos GLP-1 y demostró reducción de la aparición de nuevos eventos cardiovasculares fue el SUSTAIN 6 con la droga semaglutide. Se incluyeron 2.735 pacientes diabéticos tipo 2 con ECV establecida, enfermedad renal crónica o ambas asignados a una rama tratada con semaglutide de frecuencia semanal vs placebo. A lo largo de dos años de seguimiento se observó una reducción significativa de los 
eventos compuestos de muerte cardiovascular, IAM no fatal y ACV del orden del $26 \%$. Un descenso del $26 \%$ de la tasa de infartos no fatales (ns), una reducción significativa del $29 \%$ de la frecuencia de accidente cerebrovascular y un incremento leve no significativo de la insuficiencia cardíaca con efectos neutros sobre la mortalidad global ${ }^{28}$.

Dos estudios que emplearon drogas inhibidoras de SGLT-2 demostraron una significativa reducción de eventos cardiovasculares e insuficiencia cardíaca en pacientes diabéticos tipo 2. El estudio EMPA-REG ${ }^{29}$ realizado en 7.020 pacientes con diabetes tipo 2 con alto riesgo cardiovascular, de los cuales el 99\% había padecido evento cardiovascular previo, se asignaron aleatoriamente pacientes a tratamiento con empagliflozina $10 \mathrm{~g}$, una segunda rama recibió empagliflozina 25 mg y una tercera rama placebo más el tratamiento antidiabético convencional. Los pacientes fueron rigurosamente tratados con drogas antihipertensivas y antilipemiantes de acuerdo a las guías de práctica clínica. En el grupo asignado a empagliflozina hubo una significativa reducción de la tasa de mortalidad cardiovascular (38\% menos eventos). Si bien no se obtuvo una reducción significativa de la frecuencia de infartos no fatales e incluso hubo un incremento del 18\% de la tasa de accidentes cerebrovasculares, la reducción de la mortalidad cardiovascular se mantuvo inferior con respecto al grupo placebo. La frecuencia de hospitalización por insuficiencia cardíaca se redujo un 35\% y la mortalidad por cualquier causa fue $32 \%$ menor en el grupo asignado a empagliflozina.

En un análisis posterior del mismo estudio se incluyó una población de diabéticos con clearance de creatinina promedio de $59 \mathrm{ml} / \mathrm{min}$ o menor determinado por MDRD. El punto final primario en este segundo estudio fue un compuesto de eventos renales que incluían la duplicación de la creatinina sérica, la iniciación de terapia de reemplazo renal o la muerte por causa renal. Se observó que el grupo asignado a empagliflozina tuvo una reducción significativa de los eventos renales compuestos del or- den del 39\% con respecto a la rama placebo y la progresión a macroalbuminuria fue 38\% inferior y la duplicación de la creatinina ocurrió un $44 \%$ menos en la rama asignada a empagliflozina con respecto al placebo. La necesidad de iniciar terapia de reemplazo renal fue reducida en un 55\% en el grupo asignado a empagliflozina aunque se observaron tres muertes de causa renal en este grupo con ninguna relación en el grupo placebo. Se evidenció una leve reducción del clearance de creatinina en el período inicial de cuatro semanas en la rama tratada con empagliflozina pero posteriormente, en el largo plazo de tres años de duración, la función renal se mantuvo estable en este grupo mientras que se constató un descenso significativamente progresivo de la misma en el grupo asignado a placebo. La conclusión de los autores fue que en pacientes con diabetes tipo 2 con alto riesgo de eventos cardiovasculares el uso de empagliflozina se asoció a una progresión más lenta de la enfermedad renal con respecto al placebo ${ }^{30}$.

El estudio CANVAS ${ }^{31}$ con canagliflozina resultó del compuesto de dos estudios: el primero que se llevó a cabo entre 2009 hasta 2012 a fin de evaluar la seguridad cardiovascular de la droga y obtener la autorización de la FDA tuvo como objetivos primarios la reducción de eventos cardiovasculares compuestos (infarto no fatal, ACV y muerte cardiovascular), y el segundo en el que se colocaron como puntos finales la reducción de eventos renales esencialmente la progresión de microalbuminuria a macroalbuminuria (estudio CANVAS R). Se asignó al azar una rama canagliflozina 150 mg, canagliflozina 300 mg o placebo. En el estudio CANVAS R los pacientes que habían iniciado con 150 mg de canagliflozina podían incrementar la dosis a 300 mg. Al final del estudio, con un período de observación de seis años, se obtuvo una reducción de eventos cardiovasculares compuestos (infarto de miocardio no fatal, accidente cerebrovascular y muerte cardiovascular) del 14\% en el grupo asignado a canagliflozina. Hubo una reducción no significativa de muer- 
te por todas las causas, una disminución no significativa de la mortalidad cardiovascular y de los eventos individuales de infarto de miocardio no fatal y accidente cerebrovascular. Se observó una reducción manifiesta de los eventos de hospitalización por insuficiencia cardíaca $(27 \%)$ y una franca reducción de la progresión de microalbuminuria a macroalbuminuria del orden del $27 \%$. En este estudio se observó un incremento casi del doble del riesgo de amputaciones infrarotulianas, especialmente en dedos y metatarso, en los pacientes asignados a la rama canagliflozina, aunque en números absolutos éstos fueron de escasa magnitud (unos 6,3/1.000 pacientes por año vs 3,4/1.000 pacientes por año en el grupo placebo). En la Tabla 2 se resumen los resultados de los estudios citados.

\begin{tabular}{|c|c|c|c|c|}
\hline & LEADER & SUSTAIN-6 & EMPAREG & CANVAS \\
\hline Número de pacientes & 9.340 & 3.297 & 7.020 & 9.734 \\
\hline Seguimiento & 3,8 años & 2,1 años & 3,1 años & 3,9 años \\
\hline$\Delta \mathrm{HbA1c}$ & $-0,40 \%$ & $-1,1 / 1,4 \%$ & $-0,54 /-0,60 \%$ & $-0,58 \%$ \\
\hline$\Delta$ TA sistólica & $-1,2 \mathrm{mmHg}$ & $-3,4 / 5,4 \mathrm{mmHg}$ & $-4 \mathrm{mmHg}$ & $-3,93 \mathrm{mmHg}$ \\
\hline$\Delta$ Peso & $-2,3 \mathrm{~kg}$ & $-3,6 /-4,9 \mathrm{~kg}$ & $-1,8 /-2,2 \mathrm{~kg}$ & $-1,6 \mathrm{~kg}$ \\
\hline $\begin{array}{l}\text { Reduccion de eventos CV } \\
\text { compuestos }\end{array}$ & $-13 \%(p: 0,007)$ & $\begin{array}{l}-26 \% \\
(p<0,001)\end{array}$ & $\begin{array}{l}-14 \% \\
(p: 0,04)\end{array}$ & $\begin{array}{l}-14 \% \\
(p<0,0001)\end{array}$ \\
\hline Muerte cardiovascular & $\begin{array}{l}-22 \% \\
\text { (p:0,007) }\end{array}$ & $-2 \%(\mathrm{~ns})$ & $\begin{array}{l}-38 \% \\
(p<0,001)\end{array}$ & $-13 \%(\mathrm{~ns})$ \\
\hline IAM no fatal & $-14 \%(n s)$ & $-26 \%(n s)$ & $-13 \%(\mathrm{~ns})$ & $-15 \%(\mathrm{~ns})$ \\
\hline ACV & $14 \%(\mathrm{~ns})$ & $39 \%(p: 0,04)$ & $+18 \%(\mathrm{~ns})$ & $-10 \%(\mathrm{~ns})$ \\
\hline Insuficiencia cardíaca & $-13 \%(\mathrm{~ns})$ & $+11 \%$ (ns) & $-35 \%(\mathrm{p}: 0,002)$ & $-33 \%(\mathrm{~ns})$ \\
\hline Muerte por cualquier causa & $-15 \%(\mathrm{p}: 0,02)$ & $+0,05 \%(\mathrm{~ns})$ & $32 \%(<0,001)$ & $13 \%(\mathrm{~ns})$ \\
\hline
\end{tabular}

Tabla 2: Ensayos clínicos randomizados con drogas hipoglucemiantes orales que han demostrado reducción de eventos cardiovasculares compuestos y de mortalidad cardiovascular.

\section{BIBLIOGRAFÍA}

1. Cardiovascular disease and risk management. Standars of medical care. Diabetes Care 2017; 40 (Suppl 1):S57-S87.

2. Chobanian A. Hypertension in 2017: what is the right target? JAMA 2017; 317(6):579-580.

3. The ACCORD study group. N Engl J Med 2010; 362:15751585.

4. Mc Brien K, Rabi DM, Campbell N, et al. Intensive and standard blood pressure targets in patients with type 2 diabetes mellitus: systematic review and meta-analysis. Arch Intern Med 2012; 172(17):1296-1303.

5. Lewis EJ, Hunsicker LG, Bain RP, Rohde RD. The effect of angiotensin-converting-enzyme inhibition on diabetic nephropathy. The Collaborative Study Group. The Collaborative study group. N Engl J Med 1993; 329(20):1456-62.

6. Effects of ramipril on cardiovascular and microvascular outcomes in people with diabetes mellitus: results of the HOPE study and MICRO-HOPE substudy. Heart Outcomes Prevention Evaluation Study Investigators. Lancet 2000; 355 (9200):253-9.

7. Brenner BM, Cooper ME, de Zeew D, et al. Effects of losartan on renal and cardiovascular outcomes in patients with type 2 diabetes and nephropathy. N Engl J Med 2001; 345: 861-869.
8. Lewis EJ , Hunsincker LG, Clarke WH, BerlT, et al. Renoprotective effect of the angiotensin-receptor antagonist irbesartan in patients with nephropathy due to type 2 diabetes. N Engl J Med 2001; 345:851-860.

9. Parving $\mathrm{HH}$, Lehnert $\mathrm{H}$, Bröchner-Mortensen $\mathrm{J}$, et al. The microalbuminuric study group. The effects of irbersartan on the development of diabetic nephropathy in patients with type 2 diabetes. N Engl J Med 2001; 345: 870-878.

10. The ONTARGET investigators. Telmisartan, ramipril, or both in patients at high risk of vascular events. N Engl J Med 2008; 358:1547-49.

11. The VA NEPHRON investigators. Combined angiotensin inhibition for the treatment of diabetic nephropathy. N Engl J Med 2013; 369:1892-903.

12. Cheng J, Zhang W, Zhang X, et al. Effects of angiotensin converting enzyme inhibitors and angiotensin II receptor bloquers on all cause mortality, cardiovascular deaths and cardiovascular events in patients with diabetes mellitus: a meta-analysis. JAMA Intern Med 2014; 174(5)773-85.

13. Hao G, Wang Z, Guo R, et al. Effects of ACEl/ARB in hypertensive patients with type 2 diabetes mellitus: a meta-analisis of randomized controlled studies. BMC Cardiovasc Disord 2014; 14:148. 
14. McMurray JJ, Milton Packer M, Desai S; The PARADIGM HF investigators. Angiotensin-neprilysin inhibition vs enalapril in heart failure. N Engl J Med 2014; 371:993-1004.

15. Seferovic JP, Claggett B, Seidelman SB, et al. Effects of sacubitril/valsartan vs enalapril on glycemic control in patients with heart failure and diabetes: a post hoc analysis from the PARADIGM-HF trial. Lancet Diabetes Endocrinol 2017; 5 (5):333-340.

16. Jamerson K, Weber M, Bakris G; The ACCOMPLISH trial investigators. Benazepril plus amlodipine or hydroclorothiazide for hypertension in high risk patients. N Engl J Med 2008; 359(4)2417-28.

17. Dahlof B, Server PS, Poulter NR, et al. The Ascot investigators: Prevention of cardiovascular events with an antihypertensive regimen of amlodipine adding perindopril as required vs atenolol adding bedroflumethiazide as required in the Anglo Scandinavian Cardiac OutcomesTrial Blood pressure lowering arm (ASCOT-BPA): a multicenter randomised controled trial. Lancet 2005; 366 (9489):895-906.

18. Lindholm LH, Ibsen H, Dahlof B; The LIFE Study group. Cardiovascular morbidity and mortality in patients with diabetes in the losartan intervention for end point reduction in hypertension study (LIFE): A randomised trial against atenolol. Lancet 2002; 359 (9311):1004-10.

19. UK prospective study group. Tight blood pressure and risk of macrovascular and microvascular complications in type 2 diabetics. BMJ 1998; 317:703-713.

20. Hansson L, Lindholm LH,Niskanen L, et al. Effect of angiotensin-converting-enzyme inhibition compared with conventional therapy on cardiovascular morbidity and mortality in hypertension: the Captopril Prevention Project (CAPPP) randomised trial. Lancet 1999; 353(9153):611-6.

21. Bakris G, Fonseca V, Katholi, et al. Metabolic effect of carvedilol vs metoprolol in patients with type 2 diabetes meIlitus and hypertension. JAMA 2004; 292(18):2227-2583.
22. Nissen SE, Wolsky K. Effect of rosiglitazone on the risk of myocardial infarction and death from cardiovascular causes. N Engl J Med 2007; 356:2457-71.

23. Food and Drug Administration U.S Department of Health and Human Services. Center of Drug Evaluation and Research. December 2008: Guidance for industry. Diabetes mellitus: evaluating cardiovascular risk in new antidiabetic therapies to treat type 2 diabetes.

24. Green JB, Bethel MA, Armstrong PW, et al.; The Tecos Study group. Effect of sitagliptin on cardiovascular outcomes in type 2 diabetes. N Engl J Med 2015; 373:232-42.

25. Scirica B, Bhatt D, Banwald E, Steg G, Davidson J, Hirshberg B; the SavorTimi Steering Committe and Investigators. Saxagliptin and cardiovascular outcomes in patients with type 2 diabetes mellitus. N Engl J Med 2013; 369:1317-1326.

26. Filion K, Azoulay L, Platt R, et al; the CNODES Investigators. A multicenter observational study of incretin based drugs and heart failure. N Engl J Med 2016; 374:11451154.

27. Marso SP, Daniels GH, Brown-Frandsen K; the Leader Steering Committee. Liraglutide and cardiovascular outcomes in type 2 diabetes. N Engl J Med 2016; 375:311-322.

28. Marso SP, Brain SC, Consoli A, et al. Semaglutide and cardiovascular outcomes in patients with type 2 diabetes. N Engl J Med 2016; 375:1834-44.

29. Zinman B, Wanner Ch, Lachin J, et al. Empaglifozin, cardiovascular outcomes and mortality in type 2 diabetes. N Engl J Med 2015; 373(22):2117-2128.

30. Wanner Ch, Inzucchi S, Lachi, et al. Empaglifozin and progession of kidney disease in type 2 diabetes. $\mathrm{N}$ Engl J Med 2016; 37:323-334.

31. Neal B, Perkovic V, Mahaffey KW, et al. Canaglifozin and cardiovascular and renal events in type 2 diabetes. $\mathrm{N}$ Engl J Med jun 2017( en impresión). 